HEPHY-PUB 703/98

UWThPh-1998-58

October 1998

\title{
SOLVING THE SCHRÖDINGER EQUATION FOR BOUND STATES WITH MATHEMATICA 3.0
}

\author{
Wolfgang LUCHA \\ Institut für Hochenergiephysik, \\ Österreichische Akademie der Wissenschaften, \\ Nikolsdorfergasse 18, A-1050 Wien, Austria \\ Franz F. SCHÖBERL \\ Institut für Theoretische Physik, \\ Universität Wien, \\ Boltzmanngasse 5, A-1090 Wien, Austria
}

\begin{abstract}
Using Mathematica 3.0, the Schrödinger equation for bound states is solved. The method of solution is based on a numerical integration procedure together with convexity arguments and the nodal theorem for wave functions. The interaction potential has to be spherically symmetric. The solving procedure is simply defined as some Mathematica function. The output is the energy eigenvalue and the reduced wave function, which is provided as an interpolated function (and can thus be used for the calculation of, e.g., moments by using any Mathematica built-in function) as well as plotted automatically. The corresponding program schroedinger.nb can be obtained from franz.schoeberl@univie.ac.at.
\end{abstract}




\section{Introduction}

The Schrödinger equation is one of the fundamental equations (of motion) in physics. Unfortunately, exact analytic solutions may be found only in exceptional cases. A wide area of application has been and still is nonrelativistic potential models, which describe bound-state properties of hadrons, considered as bound states of quarks interacting via some spherically symmetric potential. Detailed discussions may be found in Refs. [1, 2]. Fortunately, the two-body Schrödinger equation with spherically symmetric potential $V(r)$, where $r \equiv|\mathbf{x}|$ and $\mathbf{x}$ is the relative coordinate of the constituents, can be reduced to an ordinary differential equation for the reduced wave function $y_{n, \ell}(r)$, describing a bound state of radial and orbital-angular-momentum quantum numbers $n$ and $\ell$, resp. In natural units, where $\hbar=c=1$, the (nonrelativistic) two-body Schrödinger equation in configuration space for the reduced wave function, which is normalized according to

$$
\int_{0}^{\infty} \mathrm{d} r\left[y_{n, \ell}(r)\right]^{2}=1
$$

reads

$$
\left[\frac{1}{2 \mu}\left(-\frac{\mathrm{d}^{2}}{\mathrm{~d} r^{2}}+\frac{\ell(\ell+1)}{r^{2}}\right)+V(r)\right] y_{n, \ell}(r)=E_{n, \ell} y_{n, \ell}(r) .
$$

$\ell=0,1,2, \ldots$ denotes the angular-momentum quantum number, $\mu$ the reduced mass,

$$
\mu \equiv \frac{m_{1} m_{2}}{m_{1}+m_{2}}
$$

and $E_{n, \ell}$ is the energy eigenvalue, with $n=0,1, \ldots$ counting the number of nodes of the bound-state wave function within $(0, \infty)$, which corresponds to the radial excitations.

Here, a Mathematica [3] notebook is constructed for the numerical solution of the reduced Schrödinger equation. The solution of differential equations with Mathematica consumes more computational time than with Fortran since the Mathematica program is not compiled. On the other hand, it is rather complicated to handle graphics within Fortran. Moreover, using Fortran one has to write a program for any new potential, to compile it, and to link it. Thus, it is much more tedious to study various potentials with Fortran than with Mathematica. In Mathematica, it is rather easy to define a function, to calculate matrix elements, and to use graphics tools.

The outline of this paper is to discuss first the method of finding the solutions, then to demonstrate the application of Mathematica, and, finally, to give, in the Appendix, the program listing. (It may be obtained as Mathematica notebook schroedinger.nb from franz.schoeberl@univie.ac.at.) The method applied here is based on Ref. [4].

\section{Method of Solution}

Rewriting Eq. (2) in more convenient form, the differential equation to be integrated is

$$
y_{n, \ell}^{\prime \prime}(r)=\left[V_{\mathrm{eff}}(r)-\varepsilon_{n, \ell}\right] y_{n, \ell}(r),
$$

with the effective potential

$$
V_{\text {eff }}(r) \equiv 2 \mu V(r)+\frac{\ell(\ell+1)}{r^{2}}
$$

and the scaled energy eigenvalue

$$
\varepsilon_{n, \ell} \equiv 2 \mu E_{n, \ell}
$$


For the energy eigenvalue $E$ to be bounded from below, the potential $V(r)$ has to be, in any case, less singular than $-1 / r^{2}$. Moreover, for potentials $V(r)$ such that $r^{2} V(r)$ is analytic (which implies that $V(r)$ is less singular than $1 / r^{2}$ ), the general (normalizable) solution of the differential equation (3) is given as a power-series expansion of the form

$$
y(r) \propto r^{\ell+1}[1+O(r)] .
$$

The asymptotic behaviour of the not normalized reduced wave function $y(r)$ for $r \rightarrow 0$ is therefore

$$
\lim _{r \rightarrow 0} y(r)=r^{\ell+1}
$$

The normalization condition (1) forces the reduced wave function $y(r)$ to approach zero when $r \rightarrow \infty$. Consequently, the main idea of finding the energy eigenvalues $E$ and corresponding reduced wave functions $y$ is to perform a systematic scan for increasing values of $\varepsilon$ in Eq. (3), looking for those values of $\varepsilon$ which allow $y(r) \rightarrow 0$ for $r \rightarrow \infty$. Without loss of generality, near the origin $y$ may be assumed to be positive: $y(0+) \geq 0$.

- For $\varepsilon$ low enough, $V_{\text {eff }}(r)-\varepsilon$ is certainly positive and thus $y(r) \rightarrow+\infty$ for $r \rightarrow \infty$.

- Increasing $\varepsilon$, the divergence of $y(r)$ for $r \rightarrow \infty$ will weaken.

- Increasing $\varepsilon, V_{\text {eff }}(r)-\varepsilon$ will become negative in certain regions of $r$. If this region is large enough, it may happen that $y(r)$ vanishes for $r \rightarrow \infty$ (cf. Fig. 1): the lowest bound-state energy $E_{0, \ell}$ for given $\ell$ has been found.

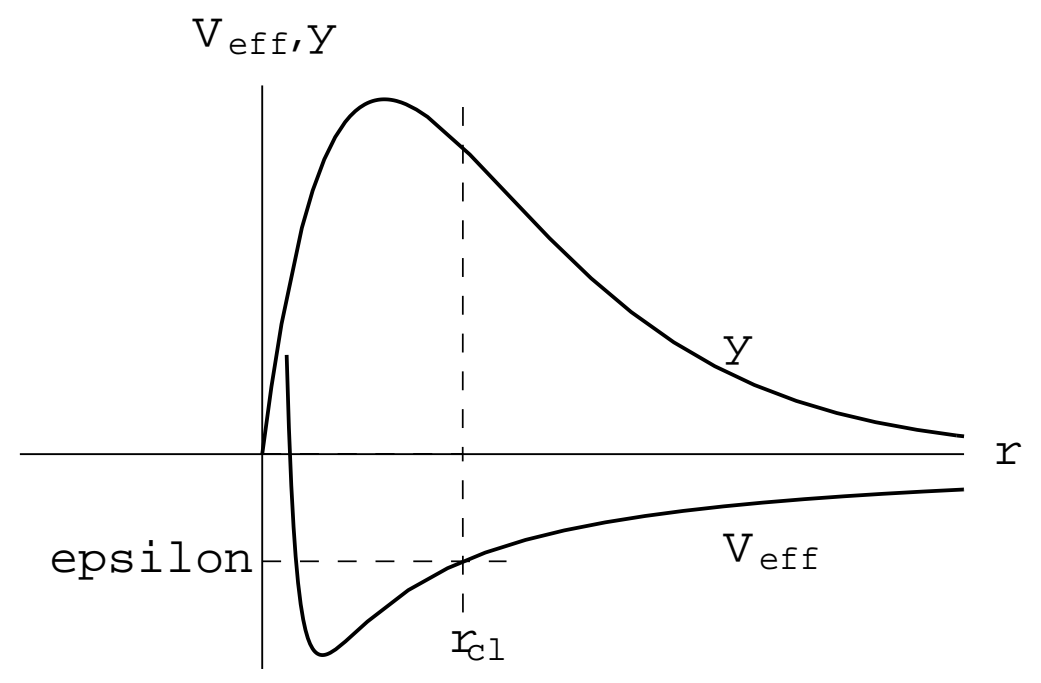

Figure 1: Qualitative behaviour of potential $V_{\mathrm{eff}}(r)$ and reduced ground-state wave function $y(r)$

- Increasing $\varepsilon, y(r)$ will cross 0 somewhere and behave like $y(r) \rightarrow-\infty$ for $r \rightarrow \infty$.

- Increasing $\varepsilon$ further, it may happen that $y(r)$ approaches 0 from below for $r \rightarrow \infty$ : the energy eigenvalue $E_{1, \ell}$ of the first radial excitation for given $\ell$ has been found.

In more detail our procedure works as follows: In principle, the integration of Eq. (3) starts at the origin. For singular potentials, however, it has to start at some value $r=\delta$ close to but different from the origin and to respect, of course, the boundary conditions

$$
\begin{aligned}
y(\delta) & =\delta^{\ell+1}, \\
y^{\prime}(\delta) & =(\ell+1) \delta^{\ell} .
\end{aligned}
$$


First, note that there is a classical turning point such that $V_{\text {eff }}(r)>\varepsilon_{n, \ell}$ for all $r>r_{\mathrm{cl}}$; the classical turning point $r_{\mathrm{cl}}$ is the largest value of $r$ solving the equation $V_{\text {eff }}(r)=\varepsilon_{n, \ell}$. Secondly, note that

$$
\operatorname{sign} y^{\prime \prime}(r)=\operatorname{sign} y(r) \quad \text { for all } r>r_{\mathrm{cl}} \text {. }
$$

This means, the reduced wave function $y$ is convex (concave) if it is positive (negative). Thus, at some point $r_{>}>r_{\mathrm{cl}}, y\left(r_{>}\right)>0$ and $y^{\prime}\left(r_{>}\right)>0$ implies $y(r) \rightarrow+\infty$ for $r \rightarrow \infty$ while $y\left(r_{>}\right)<0$ and $y^{\prime}\left(r_{>}\right)<0$ implies $y(r) \rightarrow-\infty$ for $r \rightarrow \infty$. Clearly, for both cases the integration can be stopped.

The level of excitation found in the course of the integration procedure is identified, according to the well-known nodal theorem, by the number $n$ of nodes of the (reduced) radial wave function: the ground-state wave function has no node at all $(n=0)$, while the wave function of some radial excitation has a finite number of nodes $(n=1,2, \ldots)$.

In order to locate a desired bound state, define an interval for the energy $\varepsilon$, by fixing appropriate lower and upper bounds $E_{\mathrm{L}}$ and $E_{\mathrm{U}}$, resp. The routine starts to integrate, with the Runge-Kutta method [5], the differential equation (3) at $r=\delta$, respecting the boundary conditions (44) and using, for the energy $\varepsilon$, the arithmetic mean $\left(E_{\mathrm{L}}+E_{\mathrm{U}}\right) / 2$. It counts the number of nodes, $n$, detected within a prescribed interval $\left(E_{\mathrm{L}}, E_{\mathrm{U}}\right)$ during the integration and changes $E_{\mathrm{L}}$ and $E_{\mathrm{U}}$ appropriately in an iterative procedure: if these bounds are chosen badly, $\varepsilon$ converges to that bound which lies next to the true energy; in order to cover nevertheless the desired bound state, the corresponding bound has to be changed.円

\section{Applications}

The notebook is called schroedinger.nb. It identifies $r_{\mathrm{cl}}$ by determination of the local minimum of the effective potential at largest $r$. For numerical reasons, it uses an upper bound on this minimum by adding one stepsize $h$ to it. Of course, for some potentials, like all pure power-law potentials, this local minimum may be determined analytically. For instance, for some potential of the form $V(r)=r^{k}, k \in N$, the minimum resides at

$$
r=\left[\frac{\ell(\ell+1)}{k \mu}\right]^{1 /(k+2)} .
$$

For more complicated potentials, the local minimum has to be determined numerically. This is done by the module xwmil1; the arguments of the latter are orbital excitation $\ell$, stepsize $h$ of the numerical integration, as well as stepsize weit and starting value xrat of the minimum search. Both $\ell$ and $h$ are taken over from the calling procedure schroe, which calls xwmil1 with stepsize weit $=0.5$ and guessed minimum at xrat=20. (These values are rather good starting values for the minimum search; they may be changed in the module schroe.)

Upon starting the notebook schroedinger.nb, one is provided with explanations of the usage as well as a description of the definitions used. The procedure goes as follows:

Input: schroedinger.nb.

Output: description of usage and definitions.

Input: potential $\mathrm{vl}\left[\mathrm{r}_{-}\right]:=\ldots$; e.g., for the harmonic-oscillator potential vl $\left[r_{-}\right]:=r^{\wedge} 2$.

\footnotetext{
${ }^{1}$ The program listing in the Appendix gives more details.
} 
Input: schroe $[0,20,2,1,0.01,1,1]$. The arguments of this routine are:

lower bound $E_{\mathrm{L}}$ on the energy eigenvalue $\varepsilon$ in chosen units of energy $(=0)$, upper bound $E_{\mathrm{U}}$ on the energy eigenvalue $\varepsilon$ in chosen units of energy $(=20)$, number $n$ of nodes $=$ radial excitations $(=2)$, angular momentum $\ell=$ orbital excitations $(=1)$, stepsize $h$ in chosen units of energy $(=0.01)$, masses $m_{1}, m_{2}$ in chosen units of energy $\left(m_{1}=m_{2}=1\right)$.

Output: energy eigenvalue $E_{n, \ell}$ in chosen units of energy, etc.

The above example generates as output the seeked numerical results and - if desireda plot of the (not normalized) reduced wave function $y$ (here called yschr) as function of $r$ (here called $\mathrm{x}$ ):

$\mathrm{E}=12.99999714, \mathrm{~L}=1, \mathrm{~N}=2$, Integration steps $=589, \mathrm{~h}=0.01, \mathrm{del}=0.001, \mathrm{el}=0, \mathrm{eu}=20$, Largest $\mathrm{x}$, upper integration limit, $\mathrm{XMAX}=5.881$, Smallest $\mathrm{x}$, lower integration limit, XMIN $=0.001$.

The not normalized reduced wave function is yschr[x]. The normalization factor is given by: $1 /$ NIntegrate $\left[y \operatorname{sch} r[\mathrm{x}]^{\wedge} 2,\{\mathrm{x}, \operatorname{del}, \mathrm{xmax}\}\right]$

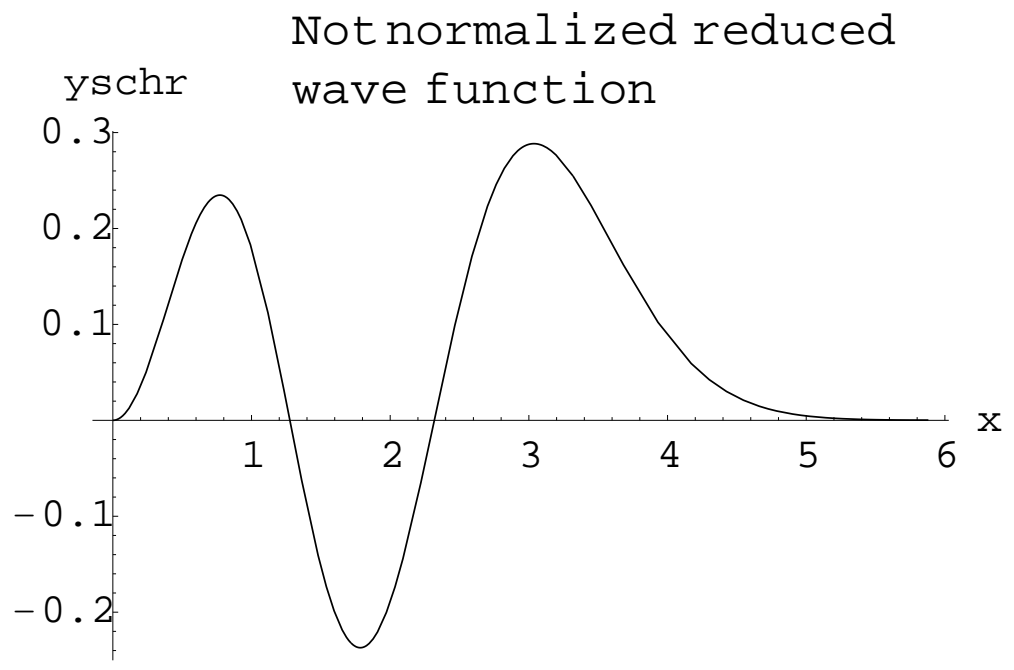

We are now able to use Mathematica built-in functions to evaluate matrix elements like $\langle 1 / r\rangle$.

Input: NIntegrate $\left[1 / \mathrm{r}\right.$ yschr $\left.[r]^{\wedge} 2,\{r, \operatorname{del}, \operatorname{xmax}\}\right] /$ NIntegrate $[y \operatorname{sch} r[r] \wedge 2,\{r, \operatorname{del}, \operatorname{xmax}\}]$.

Output: 0.625982 .

Table 1 lists results of a test run for the harmonic-oscillator potential while Table 2 shows results for the Coulomb potential for varying stepsizes $h$ and starting points del.

Obviously, $y(0)$ has to vanish for all states while $y^{\prime}(0)$ is nonvanishing only for $\ell=0$ states - which provides a (trivial) additional consistency check. Of course, the required computational time depends strongly on the desired accuracy.

In summary, the Mathematica notebook developed here provides an easy-to-handle procedure for computing energies and wave functions of (nonrelativistic) bound states. 
Table 1: Energy eigenvalues, moments, as well as wave function and its derivative at the origin for the harmonic-oscillator potential $V(r)=r^{2}$. The values chosen for the parameters are $m_{1}=m_{2}=1 \mathrm{GeV}$, $h=0.01 \mathrm{GeV}^{-1}, E_{\mathrm{L}}=0 \mathrm{GeV}$, and $E_{\mathrm{U}}=20 \mathrm{GeV}$. The exact energies are $E_{n, \ell}=4 n+2 \ell+3 \mathrm{GeV}$; the discrepancies of the computed values only reflect the $h$-dependent accuracy of our numerical method.

\begin{tabular}{ccrcccc}
\hline \hline$n$ & $\ell$ & $E_{n, \ell}[\mathrm{GeV}]$ & $\langle 1 / r\rangle[\mathrm{GeV}]$ & $\left\langle 1 / r^{2}\right\rangle\left[\mathrm{GeV}^{2}\right]$ & {$[y(0)]^{2}[\mathrm{GeV}]$} & {$\left[y^{\prime}(0)\right]^{2}\left[\mathrm{GeV}^{3}\right]$} \\
\hline 0 & 0 & 2.999997 & 1.1284 & 1.9977 & 0.0000 & 2.2567 \\
1 & 0 & 7.000003 & 0.9403 & 1.9966 & 0.0000 & 3.3851 \\
2 & 0 & 10.999999 & 0.8369 & 1.9958 & 0.0000 & 4.2314 \\
0 & 1 & 4.999995 & 0.7523 & 0.6667 & 0.0000 & 0.0000 \\
1 & 1 & 9.000001 & 0.6770 & 0.6667 & 0.0000 & 0.0000 \\
2 & 1 & 12.999997 & 0.6260 & 0.6667 & 0.0000 & 0.0000 \\
0 & 2 & 7.000003 & 0.6018 & 0.4000 & 0.0000 & 0.0000 \\
1 & 2 & 10.999999 & 0.5588 & 0.4000 & 0.0000 & 0.0000 \\
2 & 2 & 15.000005 & 0.5266 & 0.4000 & 0.0000 & 0.0000 \\
\hline \hline
\end{tabular}

Table 2: Energy eigenvalues, moments, and derivative of the wave function at the origin for the 1S, 2S, and $2 \mathrm{P}$ states of the Coulomb potential $V(r)=-1 / r$ as function of the stepsize $h$. The starting point $\delta$ is calculated from $\delta=h / 10$; the numerical values chosen for the parameters are $m_{1}=m_{2}=1 \mathrm{GeV}$, $E_{\mathrm{L}}=-1 \mathrm{GeV}$, and $E_{\mathrm{U}}=+1 \mathrm{GeV}$. The corresponding exact results are given in parentheses.

\begin{tabular}{|c|c|c|c|c|c|c|}
\hline State & $1 \mathrm{~S}$ & & $2 \mathrm{~S}$ & & $2 \mathrm{P}$ & \\
\hline$h\left[\mathrm{GeV}^{-1}\right]$ & 0.1 & 0.05 & 0.1 & 0.05 & 0.1 & 0.05 \\
\hline$-E_{n, \ell}[\mathrm{GeV}]$ & $\begin{array}{l}0.249973 \\
(0.25)\end{array}$ & 0.249996 & $\begin{array}{l}0.062496 \\
(0.0625)\end{array}$ & 0.062496 & $\begin{array}{l}0.062504 \\
(0.0625)\end{array}$ & 0.062504 \\
\hline$\langle 1 / r\rangle[\mathrm{GeV}]$ & $\begin{array}{l}0.4999 \\
(0.5)\end{array}$ & 0.4999 & $\begin{array}{l}0.1250 \\
(0.125)\end{array}$ & 0.1250 & $\begin{array}{l}0.1250 \\
(0.125)\end{array}$ & 0.1250 \\
\hline$\left\langle 1 / r^{2}\right\rangle\left[\mathrm{GeV}^{2}\right]$ & $\begin{array}{l}0.4947 \\
(0.5)\end{array}$ & 0.4974 & $\begin{array}{l}0.06185 \\
(0.0625)\end{array}$ & 0.06221 & $\begin{array}{l}0.02082 \\
(0.02083)\end{array}$ & 0.02082 \\
\hline$\left[y^{\prime}(0)\right]^{2}\left[\mathrm{GeV}^{3}\right]$ & $\begin{array}{l}0.4897 \\
(0.5)\end{array}$ & 0.4949 & $\begin{array}{l}0.06123 \\
(0.0625)\end{array}$ & 0.06190 & $\begin{array}{l}2.10^{-6} \\
(0)\end{array}$ & $4.10^{-7}$ \\
\hline
\end{tabular}

\section{References}

[1] H. Grosse and A. Martin, Phys. Reports 60 (1980) 341.

[2] W. Lucha, F. F. Schöberl, and D. Gromes, Phys. Reports 200 (1991) 127.

[3] S. Wolfram, Das Mathematica Buch: Mathematica Version 3 (Addison-Wesley-Longman, Bonn, 1997).

[4] P. Falkensteiner, H. Grosse, F. Schöberl, and P. Hertel, Comput. Phys. Comm. 34 (1985) 287.

[5] M. Abramowitz and I. A. Stegun, eds., Handbook of Mathematical Functions (Dover, New York 1964). 


\section{A Program Listing}

$(*$

Author: Franz F. Schöberl

Institute for Theoretical Physics

University of Vienna

Boltzmanng. 5

A-1090 Vienna

E-MAIL: Franz.Schoeberl@Univie.ac.at

\section{SOLVING THE SCHRÖDINGER EQUATION FOR BOUND STATES WITH MATHEMATICA 3.0}

This software is based on:

"Solving the Schrödinger Equation for Bound States" by

P. Falkensteiner, H. Grosse, Franz F. Schöberl, and P. Hertel

Computer Physics Communication 34 (1985) 287-293.

The energy and the reduced radial wave function for a bound state with given number of nodes n0 and angular momentum $l$ is calculated. The potential has to be spherically symmetric.

Usage:

el ..... lower bound of the energy,

eu ......upper bound of the energy,

n0 .....radial excitations, number of nodes,

1 ...... orbital excitations,

feh ....error on the energy, built in as feh $=0.00001$,

$\mathrm{h}$...... integration stepsize, determines the number of the integration steps

and thus the accuracy of the determined energy

$\mathrm{m} 1, \mathrm{~m} 2$..... constituent masses,

schroe[el,eu,n0,l,h,m1,m2] ..... calling procedure

The output reduced wave function (not normalized) is called $\mathrm{yschr}[\mathrm{x}]$.

The plot of the wave function is called yschrplot.

Example, harmonic oscillator:

(1) Define the potential: $\mathrm{vl}\left[\mathrm{x}_{\mathrm{f}}\right]:=\mathrm{x}^{\wedge} 2$

(2) Start solving the Schrödinger equation: schroe[-1,20,1,2,0.1,0.336,0.336]

The above procedure will solve the equation for the first radial and second orbital excitation.

The equation to be solved is ( $w i t h \hbar=c=1, \quad \mu=\frac{m_{1} m_{2}}{m_{1}+m_{2}}$ ):

$\left[\frac{1}{2 \mu}\left(-\frac{d^{2}}{d r^{2}}+\frac{\ell(\ell+1)}{r^{2}}\right)+V(r)\right] y_{n, \ell}(r), y s c h r[\mathrm{x}]$ is the not normalized $y_{n, \ell}(r)$,

$\psi_{n, \ell, m}(r)=\frac{y_{n, \ell}(r)}{r} Y_{\ell, m}(\Omega), \int_{0}^{\infty}\left(y_{n, \ell}(r)\right)^{2} d r=1$.

The accuracy can be increased by decreasing $\mathrm{h}$, this increases the number of integration steps. The higher the number of integration steps the more accurate the eigenvalues as well as the eigen functions. The reduced wave function will be plotted in addition. A measure for the accuracy is also the shape of the wave function. It should vanish for the largest numerical $\mathrm{x}$, otherwise one has to decrease $h$.

If you run schroe $[\mathrm{el}, \mathrm{eu}, \mathrm{n} 0,1, \mathrm{~h}, \mathrm{~m} 1, \mathrm{~m} 2]$ you automatically will be asked if you like to plot the reduced wave function.

$*)$

(*

xwmil1 calculates the minimum of the potential most to the right. The minimum

is called xwmin. xrat is some guessed $\mathrm{x}$-value most to the right

(here I have used $\mathrm{x}$-rat $=20$ ). wei1 is the stepsize of the minimum search

(here I have used wei1 $=0.5$ ).

*)

xwmil1[11_, h1_, wei1_, xrat1_, ww1_] :=

Module $[\{1=11, h=h 1$, weit $=$ wei1, $x r a t=$ xrat1, $w \mathrm{w}=\mathrm{ww} 1\}$, 


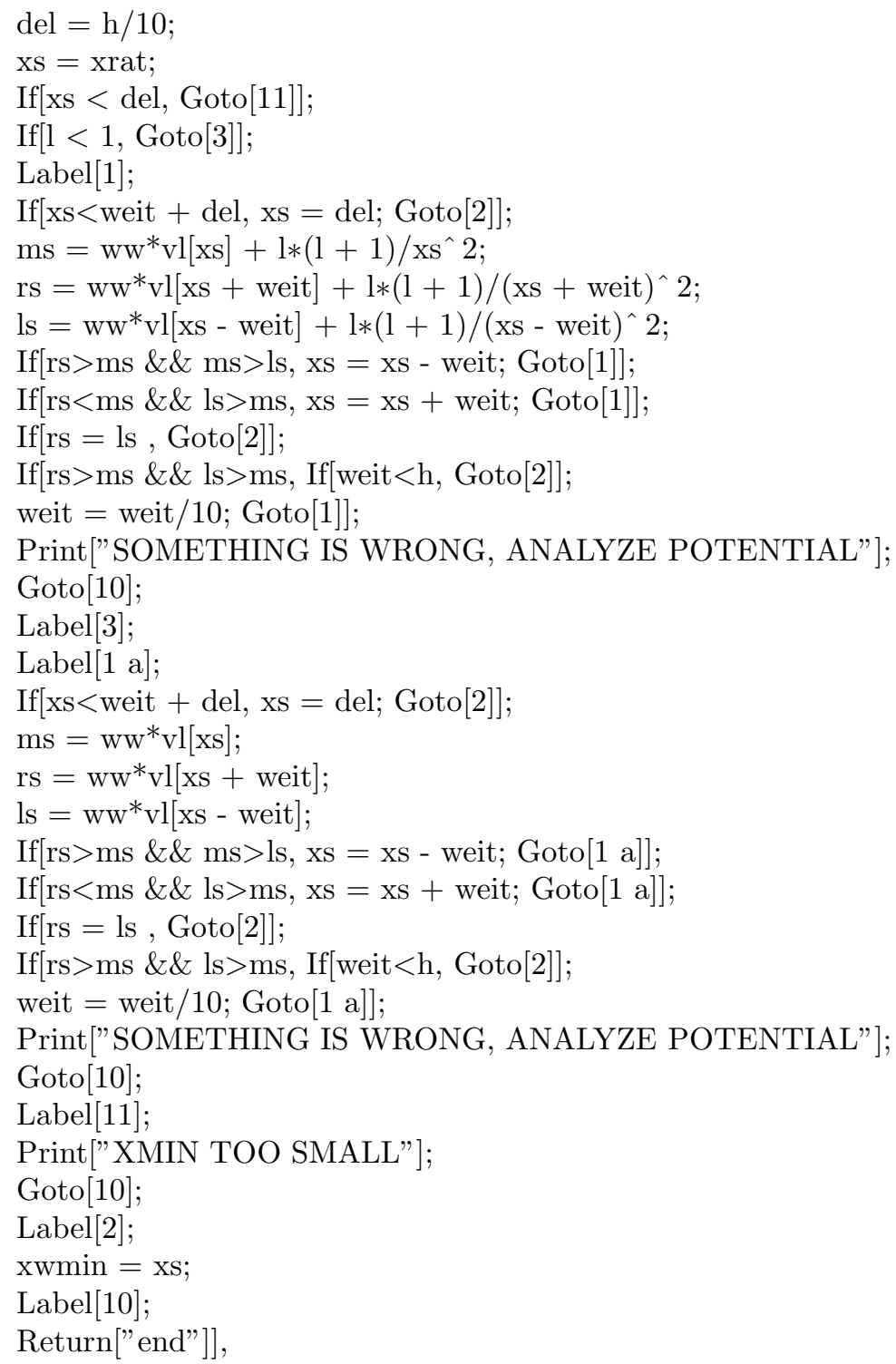




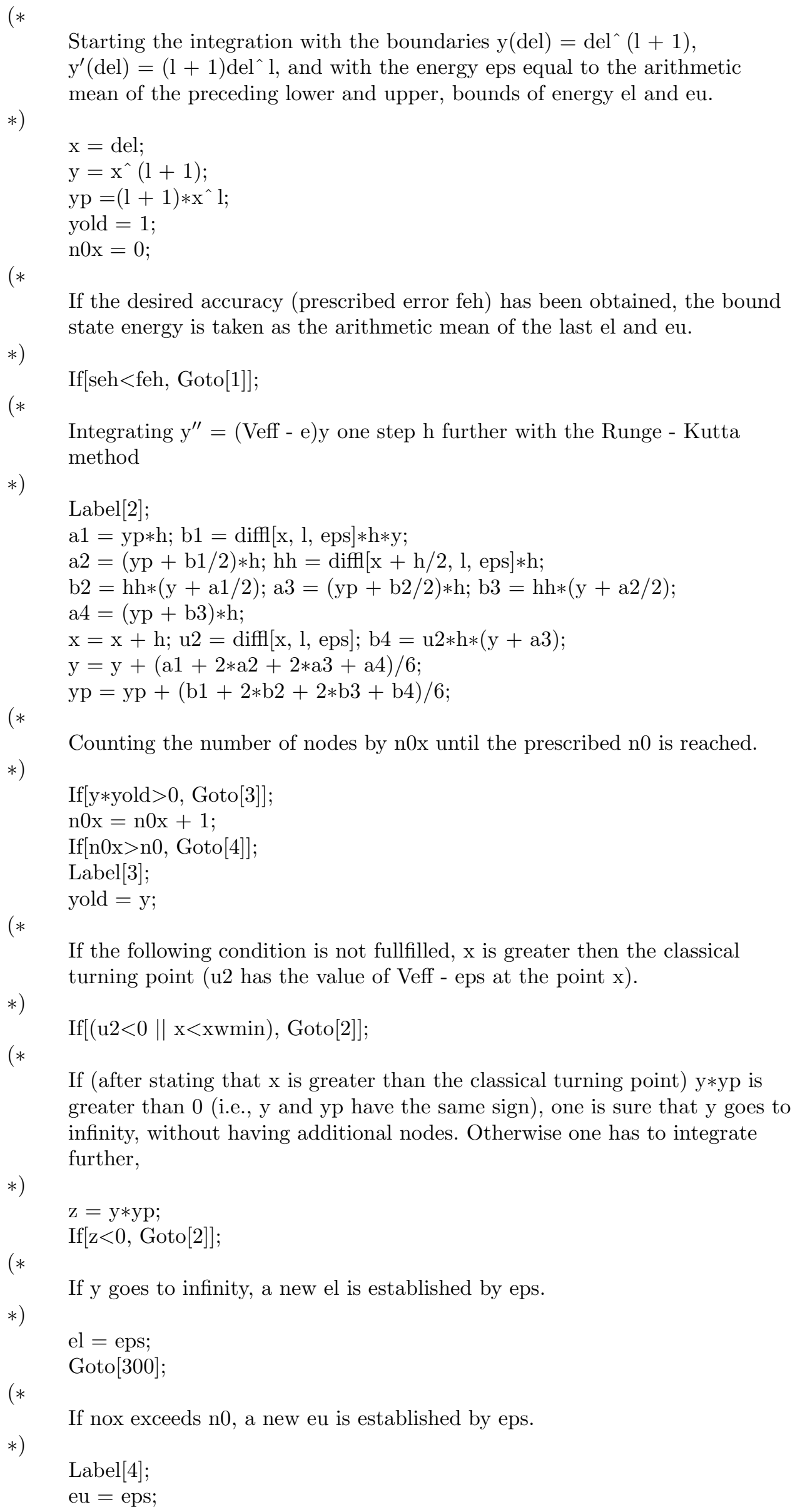

If the desired accuracy (prescribed error feh) has been obtained, the bound state energy is taken as the arithmetic mean of the last el and eu.

*)

If[seh $<$ feh, Goto[1]];

$(*$

Integrating $\mathrm{y}^{\prime \prime}=($ Veff - e)y one step h further with the Runge - Kutta method

*)

Label[2];

$\mathrm{a} 1=\mathrm{yp} * \mathrm{~h} ; \mathrm{b} 1=\operatorname{diffl}[\mathrm{x}, \mathrm{l}, \mathrm{eps}] * \mathrm{~h} * \mathrm{y}$;

$\mathrm{a} 2=(\mathrm{yp}+\mathrm{b} 1 / 2) * \mathrm{~h} ; \mathrm{hh}=\operatorname{diffl}[\mathrm{x}+\mathrm{h} / 2, \mathrm{l}, \mathrm{eps}] * \mathrm{~h} ;$

$\mathrm{b} 2=\mathrm{hh} *(\mathrm{y}+\mathrm{a} 1 / 2) ; \mathrm{a} 3=(\mathrm{yp}+\mathrm{b} 2 / 2) * \mathrm{~h} ; \mathrm{b} 3=\mathrm{hh} *(\mathrm{y}+\mathrm{a} 2 / 2)$;

$\mathrm{a} 4=(\mathrm{yp}+\mathrm{b} 3) * \mathrm{~h} ;$

$\mathrm{x}=\mathrm{x}+\mathrm{h} ; \mathrm{u} 2=\operatorname{diffl}[\mathrm{x}, \mathrm{l}, \mathrm{eps}] ; \mathrm{b} 4=\mathrm{u} 2 * \mathrm{~h} *(\mathrm{y}+\mathrm{a} 3)$;

$\mathrm{y}=\mathrm{y}+(\mathrm{a} 1+2 * \mathrm{a} 2+2 * \mathrm{a} 3+\mathrm{a} 4) / 6$

$(*$

yp $=$ yp $+(\mathrm{b} 1+2 * \mathrm{~b} 2+2 * \mathrm{~b} 3+\mathrm{b} 4) / 6$;

*)

Counting the number of nodes by n0x until the prescribed $n 0$ is reached.

If $[\mathrm{y} *$ yold $>0$, Goto[3]];

$\mathrm{n} 0 \mathrm{x}=\mathrm{n} 0 \mathrm{x}+1$;

If[n0x $>n 0$, Goto[4]];

Label[3];

yold = y;

$(*$

If the following condition is not fullfilled, $\mathrm{x}$ is greater then the classical turning point ( $\mathrm{u} 2$ has the value of Veff - eps at the point $\mathrm{x}$ ).

*)

If $[(\mathrm{u} 2<0 \| \mathrm{x}<\mathrm{xwmin})$, Goto[2]];

$(*$

If (after stating that $\mathrm{x}$ is greater than the classical turning point) $\mathrm{y} * \mathrm{yp}$ is greater than 0 (i.e., y and yp have the same sign), one is sure that y goes to infinity, without having additional nodes. Otherwise one has to integrate further,

*)

$\mathrm{z}=\mathrm{y} * \mathrm{yp}$

If $[\mathrm{z}<0$, Goto[2]];

$(*$

*)

If y goes to infinity, a new el is established by eps.

$\mathrm{el}=\mathrm{eps}$

Goto[300];

$(*$

If nox exceeds n0, a new eu is established by eps.

$*)$

Label[4];

$\mathrm{eu}=\mathrm{eps}$; 
$(*$

$*)$

$(*$

*)

$\mathrm{a} 1=\mathrm{yp} * \mathrm{~h} ; \mathrm{b} 1=\operatorname{diffl}[\mathrm{x}, \mathrm{l}, \mathrm{eps}] * \mathrm{~h} * \mathrm{y} ;$

$\mathrm{a} 2=(\mathrm{yp}+\mathrm{b} 1 / 2) * \mathrm{~h} ; \mathrm{hh}=\operatorname{diff}[\mathrm{x}+\mathrm{h} / 2, \mathrm{l}, \mathrm{eps}] * \mathrm{~h} ;$

$\mathrm{b} 2=\mathrm{hh} *(\mathrm{y}+\mathrm{a} 1 / 2) ; \mathrm{a} 3=(\mathrm{yp}+\mathrm{b} 2 / 2) * \mathrm{~h} ; \mathrm{b} 3=\mathrm{hh} *(\mathrm{y}+\mathrm{a} 2 / 2) ;$

$\mathrm{a} 4=(\mathrm{yp}+\mathrm{b} 3) * \mathrm{~h} ;$

$\mathrm{x}=\mathrm{x}+\mathrm{h} ; \mathrm{u} 2=\operatorname{diff}[\mathrm{x}, \mathrm{l}, \mathrm{eps}] ; \mathrm{b} 4=\mathrm{u} 2 * \mathrm{~h} *(\mathrm{y}+\mathrm{a} 3)$;

$\mathrm{y}=\mathrm{y}+(\mathrm{a} 1+2 * \mathrm{a} 2+2 * \mathrm{a} 3+\mathrm{a} 4) / 6$

yp $=\mathrm{y}+(\mathrm{b} 1+2 * \mathrm{~b} 2+2 * \mathrm{~b} 3+\mathrm{b} 4) / 6$;

If[y*yold $>0$, Goto[30]];

$\mathrm{n} 0 \mathrm{x}=\mathrm{n} 0 \mathrm{x}+1$

If[n0x $>$ n0, Goto[40]];

Label[30];

yold $=$ y;

If $[(\mathrm{u} 2<0 \| \mathrm{x}<\mathrm{xwmin})$, Goto[20]];

$\mathrm{z}=\mathrm{y} * \mathrm{yp}$

If $[\mathrm{z}<0$, Goto[20]];

Label[40];

$(*$

$*)$

(*

$*)$

The reduced radial wave function yschr obtained from the interpolation of the data is stored in feld1 and in xcoord

yschr $=$ Interpolation $[$ Table $[\{\operatorname{xcoord}[\mathrm{j}]$, feld $1[\mathrm{j}]\},\{\mathrm{j}, 1, \mathrm{j} 1\}]] ;$

$\operatorname{xmax}=\operatorname{xcoord}[j 1]$;

Output of the resulting energy eigenvalue and the input data.

Print[

StyleForm["E = ",

FontColor -> RGBColor[0.996109, 0, 0], FontWeight -> "Bold",

FontSize $->16]$,

StyleForm[N[ep/ww, 10],

FontColor -> RGBColor[0.996109, 0, 0], FontWeight -> "Bold",

FontSize -> 16], ",",

StyleForm["L = ",

FontColor -> RGBColor[0.996109, 0, 0], FontWeight -> "Bold",

FontSize -> 16],

StyleForm $[1$,

FontColor -> RGBColor [0.996109, 0, 0], FontWeight -> "Bold",

FontSize -> 16], ",", 
StyleForm["N = ",

FontColor -> RGBColor[0.996109, 0, 0], FontWeight -> "Bold",

FontSize ->16],

StyleForm[n0,

FontColor -> RGBColor[0.996109, 0, 0], FontWeight -> "Bold",

FontSize -> 16], ",",

Integrationsteps $=", \mathrm{j} 1, ",,, " \mathrm{~h}=", \mathrm{~h}, ",,, "$ del $="$,

del, ",", " el = ", el1, ",", " eu = ", eu1,",",

StyleForm["Largest x, upper integration limit, XMAX = ",

FontColor -> RGBColor[0, 0, 0.996109], FontWeight -> "Bold",

FontSize -> 16],

StyleForm[xmax,

FontColor -> RGBColor[0, 0, 0.996109], FontWeight -> "Bold",

FontSize -> 16], ",",

StyleForm["Smallest x, lower integration limit, XMIN = del = ",

FontColor -> RGBColor[0, 0, 0.996109], FontWeight -> "Bold",

FontSize -> 16],

StyleForm[del,

FontColor -> RGBColor[0, 0, 0.996109], FontWeight -> "Bold",

FontSize -> 16],".",

StyleForm["The reduced not normalized wave function is yschr[x].

The normalizationfactor is given by:

1/NIntegrate $\left[y \operatorname{schr}[\mathrm{x}]^{\wedge} 2,\{\mathrm{x}, \mathrm{del}, \mathrm{xmax}\}\right] "$,

FontColor -> RGBColor[0.996109, 0.500008, 0.250004],

FontWeight -> "Bold", FontSize -> 16]];

$(*$

$*)$

Preparing the plot of the reduced wave function $y \operatorname{schr}[\mathrm{x}]$

$\mathrm{zz}=$ InputString["You like to plot the (not normalized) reduced wave

function? Type yes and click OK, otherwise click just OK "];

If $[\mathrm{zz}==$ "yes", yschrplot $=\operatorname{Plot}[\mathrm{yschr}[\mathrm{x}],\{\mathrm{x}, \operatorname{del}, \mathrm{xmax}\}$,

PlotStyle -> GrayLevel[0], AxesLabel -> \{"x", "yschr" $\}$,

DefaultFont -> \{"Times-Bold", 12\},

Background -> RGBColor[0.996109, 0.996109, 0],

PlotLabel -> FontForm["Not normalized

reduced wave function", \{"Helvetica-Bold", 14\}]],

Print[StyleForm[" OK NO PLOT", FontWeight -> "Bold", FontSize -> 18,

FontColor -> RGBColor[0, 0.500008, 0]]]];

Return[]] 